\title{
Unusual Reactivity of Aliphatic and Aromatic Amines with Bromoalkyl Derivatives of Thiacalix[4]arene in 1,3-Alternate Stereoisomeric Form
}

\author{
Vladimir A. Burilov, ${ }^{a} @$ Regina R. Ibragimova, ${ }^{a}$ Bulat H. Gafiatullin, ${ }^{a}$ \\ Ramil I. Nugmanov, ${ }^{a}$ Svetlana E. Solovieva, ${ }^{\mathrm{a}, \mathrm{b}}$ and Igor S. Antipin ${ }^{\mathrm{a}, \mathrm{b}}$ \\ ${ }^{a}$ Department of Organic Chemistry, Kazan Federal University, 420008 Kazan, Russian Federation \\ bA.E. Arbuzov Institute of Organic and Physical Chemistry of Kazan Scientific Centre of Russian Academy of Sciences, \\ 420088 Kazan, Russian Federation \\ @Corresponding authorE-mail: ultrav@bk.ru
}

\begin{abstract}
For the first time a series of new cationic amphiphilic p-tert-butylthiacalix[4]arene derivatives adopting 1,3-alternate stereoisomeric form was synthesized by quaternization of several tertiary amines and N-heterocycles. The structure of all products was well-defined using modern physical technics. The influence of calixarene and amine sterical hindrances on reactivity is discussed.
\end{abstract}

Keywords: Thiacalix[4]arenes, quaternary ammonium salts, supramolecular amphiphiles.

\section{Необычная реакционная способность алифатических и ароматических аминов с бромоалкилпроизводными тиакаликс[4]арена в стереоизомерной форме 1,3-альтернат}

\author{
В. А. Бурилов, ${ }^{a}{ }^{@}$ Р. Р. Ибрагимова, ${ }^{a}$ Б. Х. Гафииатуллин, ${ }^{a}$ Р. И. Нугманов, ${ }^{a}$ \\ С. Е. Соловьева, ${ }^{a, b}$ И. С. Антипин ${ }^{a, b}$ \\ ${ }^{a}$ Кафедра органической химии Химического института им. А.М. Бутлерова, Казанский федеральньй университет, \\ 420008 Казань, Россия \\ ${ }^{\mathrm{b}}$ Институт органической и физической химии им. А.Е. Арбузова Каз НЦ РАН, 420088 Казань, Россия \\ ${ }^{\circledR}$ E-mail: ultrav@bk.ru
}

\begin{abstract}
Впервые получена серия новых катионных амфифильных производных п-трет-бутилтиакаликс[4] арена в стереоизоменой форме 1,3-альтернат путем кватернизации серии третичных аминов и азотсодержащих гетерочиклов. Структура всех производных установлена с использованием современных физических методов исследования. В статье обсуждается влияние структуры каликсарена и природы аминов на реакичионную способность.
\end{abstract}

Ключевые слова: Тиакаликс[4]арены, четвертичные аммонийные соли, супрамолекулярные амфифилы. 


\section{Introduction}

Synthetic amphiphilic host receptors with high affinity to specific guests have attracted the attention of many researchers at last decades for their wide application in supramolecular chemistry and nanotechnology in molecular recognition, drug delivery, catalysis, cell mimics, gene transfer and many other applications. ${ }^{[1-5]}$ Among all well-known macrocyclic supramolecular platforms like cyclodextrins, ${ }^{[6]}$ cucurbiturils, ${ }^{[7]}$ pillararenes, ${ }^{[8]}$ calix $[\mathrm{n}]$ arenes and their thia-analogues have a number of undeniable advantages: facile functionalization of both upper and lower rims andvariety of stereoisomeric forms. ${ }^{[9-12]}$ According to accurate expression of Seiji Shinkai, amphiphilic calixarenes are "surfactants with a host-guest recognition site". ${ }^{[13]}$

One of the most commonly used approaches for synthesis of amphiphilic calixarene derivatives is the introduction of polar groups through sulfonation ${ }^{[14]}$ or introduction of carboxylic groups. ${ }^{[15]}$ But introduction of cationic groups is in a special interest since calixarenes with cationic headgroups (i.e. amino-, quaternaryammonium, guanidine-) are privileged supramolecular amphiphiles: such cationic amphiphiles can compact DNA into lipoplexes for controlled non-viral gene delivery ${ }^{[16]}$ for the precise recognition of protein surfaces, ${ }^{[17]}$ for controlled drug delivery ${ }^{[18]}$ Moreover, calixarene-based cationic amphiphiles can act as perspective antibacterial agents towards both Gram positive and Gram negative reference bacteria. ${ }^{[19]}$

Recently ${ }^{[20]}$ we have synthesized series of cationic amphiphilic receptors by introduction of polar headgroups using copper catalyzed azide-alkyne cycloaddition reaction (CuAAC) with azide-containing thiacalix[4]arenes adopting 1,3-alternate stereoisomeric form. Use of 1,3-alternate stereoisomeric form allowed to design on the thiacalixarene platform two molecular domains (liophilic and polar receptor units) with quite different properties located on opposite sides of the macrocycles plane. Obtained cationic amphiphilic receptors showed a great opportunities in binding with biomolecules $\left(\mathrm{BSA}^{[21]}\right.$ calf timus DNA) ${ }^{[22]}$ as well as with anionic surfactants ${ }^{[23]}$ (lauril and laureth sulphates).

Herein we report the synthesis of a series of quaternary ammonium derivatives of $p$-tert-butylthiacalix[4] arene (T[4]CA) adopting 1,3-alternate stereoisomeric form with different lipophilicity through alkylation of tertiary amines or nitrogen-containing heterocycles by bis-bromine $\mathrm{T}[4]$ CA derivatives.

\section{Experimental}

All reagents were purchased from either Acros or SigmaAldrich and used without further purification. Solvents were purified according to standard methods. ${ }^{[24]}$ Parent $p$-tert-butylthiacalix[4]arene, ${ }^{[25]}$ 5,11,17,23-tetra-tert-butyl-25,27-dibutyloxy26,28-di-3-bromopropyloxy-2,8,14,20-tetrathiacalix[4]arene (1), 5,11,17,23-tetra-tert-butyl-25,27-dibutyloxy-26,28-di-4-bromobutyl oxy-2,8,14,20-tetrathiacalix[4]arene (2) and 5,11,17,23-tetra-tertbutyl-25,27-ditetradecyloxy-26,28-di-4-bromobutyloxy-2,8,14,20tetrathiacalix[4]arene (3) were synthesized according to literature methods. ${ }^{[20,26]}$

TLC was performed on Merck UV 254 plates with Vilber Lourmat VL-6.LC UV lamp (254 nm) control. Elemental analy- sis of synthesized compounds was done on the PerkinElmer PE 2400 CHNS/O Elemental Analyzer. Microwave assisted reactions were carried outinthe CEM Mars 5 Microwave Accelerated Reaction System. NMR spectra were recorded on Bruker Avance 400 Nanobay with signals from residual protons of deuterated solvents $\mathrm{CDCl}_{3}\left(\delta_{\mathrm{H}} 7.26 \mathrm{ppm}\right)$ or [D6]DMSO $\left(\delta_{\mathrm{H}} 2.50 \mathrm{ppm}\right)$ as internal standard. MALDI mass-spectra were measured on UltraFlex III TOF/ TOF with PNA matrix, laser Nd:YAG, $\lambda=355 \mathrm{~nm}$.

General procedure for synthesis of compounds 4-12. Compounds 1-3 $(0.093 \mathrm{mmol})$, ternary amine $(1.86 \mathrm{mmol})$ were dissolved in $3 \mathrm{ml}$ of dry acetonitrile. The reaction mixture was stirred for $10-45 \mathrm{~h}$ at $130{ }^{\circ} \mathrm{C}$ in 'GlassChem' vessels $\left(\mathrm{CEM}^{\circledR}\right.$ corporation) under conventional heating with TLC control (hexane:ethyl acetate=3:1). After complete of reaction solvent was evaporated in vacuo. Then $15 \mathrm{ml}$ of diethyl ether was added and the white to cream precipitate was filtered off, washed with diethyl ether $(3 \times 10 \mathrm{ml})$ and dried in vacuo.

5,11,17,23-Tetra-tert-butyl-25,27-dibutyl-26,28-bis(3-pyridinium propyloxy)-2,8,14,20-tetrathiacalix[4]arene dibromide (4). Reaction time: 37 h. Yield 0.051 g $(88 \%)$. M.p. $268^{\circ} \mathrm{C}$ (decomp.). Found (\%): C 62.53; $\mathrm{H}$ 6.90; $\mathrm{N} 2.14 ; \mathrm{S}$ 10.37. $\mathrm{C}_{64} \mathrm{H}_{84} \mathrm{O}_{4} \mathrm{~N}_{2} \mathrm{~S}_{4} \mathrm{Br}_{2}$ requires (\%): $\mathrm{C}$ 62.32; H 6.86; N 2.27; $\mathrm{S}$ 10.40. MALDI TOF $m / z: 1320[\mathrm{M}+\mathrm{Br}]^{+}$. IR (KBr) $v_{\max } \mathrm{cm}^{-1}: 3420\left(\mathrm{C}_{\mathrm{Ar}}-\mathrm{H}\right), 2961(\mathrm{C}-\mathrm{H})$, $1633(\mathrm{C}=\mathrm{N}), 1266\left(\mathrm{C}_{\mathrm{Ar}} \mathrm{O}\right) .{ }^{1} \mathrm{H} \mathrm{NMR}(400 \mathrm{MHz},[\mathrm{D} 6] \mathrm{DMSO}$, $298 \mathrm{~K}) \delta_{\mathrm{H}}$ ppm: $0.82\left(6 \mathrm{H}\right.$, br.s, $\left.\mathrm{CH}_{3}\right), 0.96-1.28\left(44 \mathrm{H}, \mathrm{m}, \mathrm{CMe}_{3}\right.$, $\left.\mathrm{CMe}_{3}, \mathrm{CH}_{2}\right), 1.75-1.85\left(4 \mathrm{H}, \mathrm{m}, \mathrm{CH}_{2}\right), 3.78\left(4 \mathrm{H}\right.$, br.t, $\left.\mathrm{OCH}_{2}\right), 3.93$ $\left(4 \mathrm{H}, \mathrm{t} J=7.74 \mathrm{~Hz}, \mathrm{OCH}_{2}\right), 4.74\left(4 \mathrm{H}, \mathrm{t} J=6.8 \mathrm{~Hz}, \mathrm{NCH}_{2}\right), 7.36(8 \mathrm{H}$, br.s, ArH), 8.23 (4H, t $J=6.9 \mathrm{~Hz}, \mathrm{PyrH}), 8.69(2 \mathrm{H}, \mathrm{t} J=7.7 \mathrm{~Hz}$, PyrH), 9.16 (4H, d $J=5.9 \mathrm{~Hz}, \mathrm{PyrH}) .{ }^{13} \mathrm{C}$ NMR (100.6 MHz, [D6] DMSO, $298 \mathrm{~K}) \delta_{\mathrm{C}}$ ppm: 157.16, 156.30, 146.01, 145.53, 145.41, $144.64,128.97,128.87,128.40,127.72,127.67,69.15,65.67,57.57$, $31.07,30.78,18.45,13.79$.

5,11,17,23-Tetra-tert-butyl-25,27-dibutyl-26,28-bis [3-(3-Nmethylimidazolium)propyloxy]-2,8,14,20-tetrathiacalix[4]arene dibromide (5). Reaction time: 34 h. Yield 0.1024 g (87 \%). M.p. $270{ }^{\circ} \mathrm{C}$ (decomp.). Found (\%): C 60.14; H 7.05; N 4.49; S 10.24 $\mathrm{C}_{62} \mathrm{H}_{86} \mathrm{O}_{4} \mathrm{~N}_{4} \mathrm{~S}_{4} \mathrm{Br}_{2}$ requires (\%): $\mathrm{C} 60.08 ; \mathrm{H} 6.99 ; \mathrm{N} 4.52 ; \mathrm{S} 10.35$. MALDI TOF $m / z$ : $1159[\mathrm{M}+\mathrm{Br}]^{+}, 1080[\mathrm{M}-2 \mathrm{Br}]^{+}$. IR $(\mathrm{KBr}) v_{\max }$ $\mathrm{cm}^{-1}: 3397\left(\mathrm{C}_{\mathrm{Ar}}-\mathrm{H}\right), 2960(\mathrm{C}-\mathrm{H}), 1653(\mathrm{C}=\mathrm{N}), 1265\left(\mathrm{C}_{\mathrm{Ar}}-\mathrm{O}\right) .{ }^{1} \mathrm{H}$ NMR $(400 \mathrm{MHz},[\mathrm{D} 6] \mathrm{DMSO}, 298 \mathrm{~K}) \delta_{\mathrm{H}} \mathrm{ppm}: 0.79(6 \mathrm{H}, \mathrm{t} J=7.1 \mathrm{~Hz}$, $\left.\mathrm{CH}_{3}\right), 0.94-1.20\left(26 \mathrm{H}, \mathrm{m}, \mathrm{CMe}_{3} \mathrm{CH}_{2}\right), 1.24\left(18 \mathrm{H}, \mathrm{s}, \mathrm{CMe}_{3}\right)$, 1.49-1.57 (4H, m, $\left.\mathrm{CH}_{2}\right), 3.76\left(4 \mathrm{H}, \mathrm{t} J=7.4 \mathrm{~Hz}, \mathrm{OCH}_{2}\right), 3.84-3.95$ $\left(7 \mathrm{H}, \mathrm{m}, \mathrm{OCH}_{2}, \mathrm{CH}_{3}\right), 4.25\left(4 \mathrm{H}, \mathrm{t} J=6.3 \mathrm{~Hz}, \mathrm{NCH}_{2}\right), 7.32$ $(4 \mathrm{H}, \mathrm{s}, \mathrm{ArH}), 7.35$ (4H, s, ArH), $7.67\left(2 \mathrm{H}\right.$, br.s, $\left.\mathrm{NCH}_{2}\right), 7.80(2 \mathrm{H}$, br.s, $\left.\mathrm{NCH}_{2}\right), 9.24(2 \mathrm{H}, \mathrm{s}, \mathrm{NCHN}) .{ }^{13} \mathrm{C} \mathrm{NMR}(100.6 \mathrm{MHz},[\mathrm{D} 6]$ DMSO, $298 \mathrm{~K}) \delta_{\mathrm{C}}$ ppm: $156.81,156.13,145.60,145.53,136.67$, $128.13,127.80,127.64,127.44,124.09,122.03,68.65,65.66,45.82$, $33.88,30.93,30.82,30.51,29.53,18.41,13.77$.

5,11,17,23-Tetra-tert-butyl-25,27-dibutyl-26,28-bis(4pyridiniumbutyloxy)-2,8,14,20-tetrathiacalix[4]arene dibromide (6). Reaction time: 12 h. Yield 0.0985 g (84 \%). M.p. $284{ }^{\circ} \mathrm{C}$ (decomp.). Found (\%): C 62.90; H 7.07; N 2.18; S 10.14. $\mathrm{C}_{66} \mathrm{H}_{88} \mathrm{O}_{4} \mathrm{~N}_{2} \mathrm{~S}_{4} \mathrm{Br}_{2}$ requires (\%): $\mathrm{C} 62.84 ; \mathrm{H} 7.03 ; \mathrm{N} 2.22 ; \mathrm{S} 10.17$. MALDI TOF $m / z: 1179[\mathrm{M}-\mathrm{Br}]^{+}$. IR $(\mathrm{KBr}) v_{\max } \mathrm{cm}^{-1}: 3398\left(\mathrm{C}_{\mathrm{Ar}^{-}}\right.$ $\mathrm{H}), 2961(\mathrm{C}-\mathrm{H}), 1634(\mathrm{C}=\mathrm{N}), 1266\left(\mathrm{C}_{\mathrm{Ar}} \mathrm{O}\right) .{ }^{1} \mathrm{H}$ NMR $(400 \mathrm{MHz}$, [D6]DMSO, $298 \mathrm{~K}) \delta_{\mathrm{H}}$ ppm: $0.68-0.87\left(10 \mathrm{H}, \mathrm{m}, \mathrm{CH}_{3}, \mathrm{CH}_{2}\right)$, 0.95-1.35 (40H, m, $\left.\mathrm{CMe}_{3}, \mathrm{CMe}_{3}, \mathrm{CH}_{2}\right), 1.85-2.01\left(4 \mathrm{H}, \mathrm{m}, \mathrm{CH}_{2}\right)$, $3.71-3.85\left(8 \mathrm{H}, \mathrm{s}, \mathrm{CH}_{2}, \mathrm{OCH}_{2}\right), 3.90\left(4 \mathrm{H}, \mathrm{t} J=7.90 \mathrm{~Hz}, \mathrm{OCH}_{2}\right), 4.55$ $\left(4 \mathrm{H}, \mathrm{t} J=7.77 \mathrm{~Hz}, \mathrm{NCH}_{2}\right), 7.31(4 \mathrm{H}, \mathrm{s}, \mathrm{ArH}), 7.37$ (4H, s, ArH), $8.22(4 \mathrm{H}, \mathrm{t} J=7.0 \mathrm{~Hz}, \mathrm{PyrH}), 8.66(2 \mathrm{H}, \mathrm{t} J=7.8 \mathrm{~Hz}, \mathrm{PyrH}), 9.11$ $(4 \mathrm{H}, \mathrm{d} J=5.8 \mathrm{~Hz}, \mathrm{PyrH}) .{ }^{13} \mathrm{C}$ NMR $(100.6 \mathrm{MHz},[\mathrm{D} 6] \mathrm{DMSO}$, $298 \mathrm{~K}) \delta_{\mathrm{C}}$ ppm: $157.13,156.77,145.74,145.26,145.16,144.78$, $128.74,128.64,128.20,127.77,127.68,69.03,68.29,59.98,33.85$, $31.08,30.96,30.82,27.12,24.87,18.49,13.81$.

5,11,17,23-Tetra-tert-butyl-25,27-dibutyl-26,28-bis[4-(3-Nmethylimidazolium)butyloxy]-2,8,14,20-tetrathiacalix[4]arene dibromide (7). Reaction time: 10 h. Yield 0.1222 g (85 \%). M.p. 
$272{ }^{\circ} \mathrm{C}$ (decomp.). Found (\%): C 60.77; H 7.21; N 4.38; S 10.08 . $\mathrm{C}_{64} \mathrm{H}_{90} \mathrm{O}_{4} \mathrm{~N}_{4} \mathrm{~S}_{4} \mathrm{Br}_{2}$ requires (\%): $\mathrm{C} 60.65 ; \mathrm{H} 7.16 ; \mathrm{N} 4.42 ; \mathrm{S} 10.12$. MALDI TOF $m / z$ : $1187[\mathrm{M}-\mathrm{Br}]^{+}, 1105[\mathrm{M}-2 \mathrm{Br}]^{+}, 1025[\mathrm{M}-2 \mathrm{Br}-$ $\left.\mathrm{C}_{4} \mathrm{H}_{6} \mathrm{~N}_{2}\right]^{+}$. IR $(\mathrm{KBr}) v_{\max } \mathrm{cm}^{-1}: 3398\left(\mathrm{C}_{\mathrm{Ar}}-\mathrm{H}\right), 2962(\mathrm{C}-\mathrm{H}), 1572(\mathrm{C}=\mathrm{N})$, $1266\left(\mathrm{C}_{\mathrm{Ar}} \mathrm{O}\right) .{ }^{1} \mathrm{H}$ NMR $(400 \mathrm{MHz},[\mathrm{D} 6] \mathrm{DMSO}, 298 \mathrm{~K}) \delta_{\mathrm{H}} \mathrm{ppm}$ : $0.79\left(6 \mathrm{H}, \mathrm{t} J=7.1 \mathrm{~Hz}, \mathrm{CH}_{3}\right), 0.99-1.36\left(44 \mathrm{H}, \mathrm{m}, \mathrm{CMe}_{3}, \mathrm{CMe}_{3}\right.$, $\left.\mathrm{CH}_{2}\right), 1.44-1.63\left(4 \mathrm{H}, \mathrm{m}, \mathrm{CH}_{2}\right), 3,76\left(4 \mathrm{H}, \mathrm{t} J=7.4 \mathrm{~Hz}, \mathrm{OCH}_{2}\right)$, 3.84-3.98 (10H, m, $\left.\mathrm{OCH}_{2}, \mathrm{CH}_{3}\right), 4.24\left(4 \mathrm{H}, \mathrm{t} J=6.3 \mathrm{~Hz}, \mathrm{NCH}_{2}\right)$, $7.33(4 \mathrm{H}, \mathrm{s}, \mathrm{ArH}), 7.35(4 \mathrm{H}, \mathrm{s}, \mathrm{ArH}), 7.65(2 \mathrm{H}, \mathrm{s}, \mathrm{NCH}), 7.80$ $(2 \mathrm{H}, \mathrm{s}, \mathrm{NCH}), 9.22(2 \mathrm{H}, \mathrm{s}, \mathrm{NCHN}) .{ }^{13} \mathrm{C} \mathrm{NMR}(100.6 \mathrm{MHz},[\mathrm{D} 6]$ DMSO, $298 \mathrm{~K}) \delta_{\mathrm{C}}$ ppm: 156.84, 156.16, 145.63, 145.55, 136.69, 128.16, 127.84, 127.67, 127.46, 124.14, 122.03, 68.66, 65.67, 45.84, $35.92,33.92,30.96,30.85,30.53,29.53,18.44,13.82$.

5,11,17,23-Tetra-tert-butyl-25,27-dibutyl-26,28-bis[4-(1-Nmethylmorpholinium)butyloxy]-2,8,14,20-tetrathiacalix[4]arene dibromide (8). Reaction time: 45 h. Yield 0.0983 g (81 \%). M.p. $269{ }^{\circ} \mathrm{C}$ (decomp.). Found (\%): C 60.94; H 7.79; N 2.11; S 9.73. $\mathrm{C}_{66} \mathrm{H}_{100} \mathrm{O}_{6} \mathrm{~N}_{2} \mathrm{~S}_{4} \mathrm{Br}_{2}$ requires (\%): $\mathrm{C} 60.72 ; \mathrm{H} 7.72 ; \mathrm{N} 2.15 ; \mathrm{S} 9.82$. MALDI TOF $\mathrm{m} / \mathrm{z}: 1224[\mathrm{M}-\mathrm{Br}]^{+}, 1130\left[\mathrm{M}-2 \mathrm{Br}-\mathrm{CH}_{3}\right]^{+}, 1044$ [M-2Br$\left.\mathrm{CH}_{3}-\mathrm{NC}_{4} \mathrm{H}_{4} \mathrm{O}\right]^{+}, 988 \quad\left[\mathrm{M}-2 \mathrm{Br}-\mathrm{CH}_{3}-\mathrm{NC}_{4} \mathrm{H}_{4} \mathrm{O}-\mathrm{C}_{4} \mathrm{H}_{8}\right]^{+}$. IR $(\mathrm{KBr})$ $v_{\max } \mathrm{cm}^{-1}: 3420\left(\mathrm{C}_{\mathrm{Ar}}-\mathrm{H}\right), 2922(\mathrm{C}-\mathrm{H}), 1267\left(\mathrm{C}_{\mathrm{Ar}}-\mathrm{O}\right), 1128\left(-\mathrm{CH}_{2}-\right.$ $\left.\mathrm{O}-\mathrm{CH}_{2}-\right)$. ${ }^{1} \mathrm{H}$ NMR $(400 \mathrm{MHz},[\mathrm{D} 6] \mathrm{DMSO}, 298 \mathrm{~K}) \delta_{\mathrm{H}} \mathrm{ppm}$ : $0.81\left(6 \mathrm{H}, \mathrm{t} J=6.8 \mathrm{~Hz}, \mathrm{CH}_{3}\right), 1.09-1.31\left(44 \mathrm{H}, \mathrm{m}, \mathrm{CMe}_{3}, \mathrm{CMe}_{3}\right.$, $\left.\mathrm{CH}_{2}\right), 1.65-1.74\left(4 \mathrm{H}, \mathrm{m}, \mathrm{CH}_{2}\right), 3.13\left(6 \mathrm{H}, \mathrm{s}, \mathrm{CH}_{3}\right), 3.28-3.64$ $\left(12 \mathrm{H}, \mathrm{m}, \mathrm{NCH}_{2}, \mathrm{NCH}_{2}, \mathrm{CH}_{2}\right), 3.75-4.02\left(16 \mathrm{H}, \mathrm{m}, \mathrm{OCH}_{2}, \mathrm{OCH}_{2}\right.$, $\left.\mathrm{OCH}_{2}\right), 7.35$ (4H, s, ArH), 7.43 (4H, s, ArH). ${ }^{13} \mathrm{C} \mathrm{NMR}\left(100.6 \mathrm{MHz}^{2}\right.$, [D6]DMSO, $298 \mathrm{~K}) \delta_{\mathrm{C}}$ ppm: 145.33, 129.04, 128.34, 127.91, 127.57, 59.86, 58.88, 45.44, 34.02, 33.91, 31.21, 31.05, 30.87, 24.92, 18.58, $17.12,13.92$.

5,11,17,23-Tetra-tert-butyl-25,27-ditetradecyl-26,28-bis(4pyridiniumbutyloxy)-2,8,14,20-tetrathiacalix[4]arenedibromide(9). Reaction time: 25 h. Yield 0.1132 g (79 \%). M.p. $255^{\circ} \mathrm{C}$ (decomp.). Found (\%): C 67.04; $\mathrm{H} 8.44 ; \mathrm{N} 1.76 ; \mathrm{S}^{2} .28 . \mathrm{C}_{86} \mathrm{H}_{128} \mathrm{O}_{4} \mathrm{~N}_{2} \mathrm{~S}_{4} \mathrm{Br}_{2}$ requires (\%): C 66.99; H 8.37; N 1.82; S 8.32. MALDI TOF $m / z$ : 1459[M$\mathrm{Br}]^{+}$. IR (KBr) $v_{\max } \mathrm{cm}^{-1}: 3397\left(\mathrm{C}_{\mathrm{Ar}}-\mathrm{H}\right), 2924(\mathrm{C}-\mathrm{H}), 1633(\mathrm{C}=\mathrm{N})$, $1269\left(\mathrm{C}_{\mathrm{Ar}}-\mathrm{O}\right)$. ${ }^{1} \mathrm{H}$ NMR $(400 \mathrm{MHz}$, [D6]DMSO, $298 \mathrm{~K}) \delta_{\mathrm{H}} \mathrm{ppm}$ : $0.85\left(6 \mathrm{H}, \mathrm{t} J=6.5 \mathrm{~Hz}, \mathrm{CH}_{3}\right), 0.95-1.58\left(84 \mathrm{H}, \mathrm{m}, \mathrm{CMe}_{3}, \mathrm{CMe}_{3}\right.$, $\left.\mathrm{CH}_{2}\right), 1.85-1.99\left(4 \mathrm{H}, \mathrm{m}, \mathrm{CH}_{2}\right), 3.70-3.84\left(8 \mathrm{H}, \mathrm{m}, \mathrm{OCH}_{2}, \mathrm{CH}_{2}\right)$, $3.90\left(4 \mathrm{H}, \mathrm{t} J=7.7 \mathrm{~Hz}, \mathrm{OCH}_{2}\right), 4.54\left(4 \mathrm{H}, \mathrm{t} J=7.8 \mathrm{~Hz}, \mathrm{NCH}_{2}\right), 7.30$ (4H, s, ArH), 7.36 (4H, s, ArH), 8.22 (4H, t J=6.8 Hz, PyrH), 8.65 $(2 \mathrm{H}, \mathrm{t} J=7.6 \mathrm{~Hz}, \mathrm{PyrH}), 9.08(4 \mathrm{H}, \mathrm{d} J=5.7 \mathrm{~Hz}, \mathrm{PyrH}) .{ }^{13} \mathrm{C} \mathrm{NMR}$ $(100.6 \mathrm{MHz}$, [D6]DMSO, $298 \mathrm{~K}) \delta_{\mathrm{C}} \mathrm{ppm}: 146.27,145.38,145.26$, $129.13,129.04,128.72,128.26,128.21,128.16,111.21,62.44,60.51$, $33.27,31.78,31.57,31.38,29.66,29.51,29.20,27.60,25.58,25.35$, $22.56,22.21,14.40$.

5,11,17,23-Tetra-tert-butyl-25,27-ditetradecyl-26,28-bis[4(3-N-methylimidazolium)butyloxy]-2,8,14,20-tetrathiacalix[4]arene dibromide (10). Reaction time: 23 h. Yield 0.1079 g (75 \%). M.p. $260{ }^{\circ} \mathrm{C}$ (decomp.). Found (\%): C 65.23; H 8.52; N 3.57; S 8.20. $\mathrm{C}_{84} \mathrm{H}_{130} \mathrm{O}_{4} \mathrm{~N}_{4} \mathrm{~S}_{4} \mathrm{Br}_{2}$ requires (\%):C 65.17; $\mathrm{H}$ 8.46; N 3.62; $\mathrm{S} 8.29$. MALDI TOF $\mathrm{m} / \mathrm{z}$ : $1465[\mathrm{M}-\mathrm{Br}]^{+}$. IR $(\mathrm{KBr}) v_{\mathrm{max}} \mathrm{cm}^{-1}: 3423\left(\mathrm{C}_{-}^{-}\right.$ $\mathrm{H}), 2921(\mathrm{C}-\mathrm{H}), 1574(\mathrm{C}=\mathrm{N}), 1266\left(\mathrm{C}_{\mathrm{Ar}}-\mathrm{O}\right) .{ }^{1} \mathrm{H}$ NMR $(400 \mathrm{MHz}$, [D6]DMSO, $298 \mathrm{~K}) \delta_{\mathrm{H}}$ ppm: $0.86\left(6 \mathrm{H}\right.$, br.t, $\left.\mathrm{CH}_{3}\right), 0.95-1.34$ $\left(84 \mathrm{H}, \mathrm{m}, \mathrm{CH}_{2}, \mathrm{CMe}_{3}, \mathrm{CMe}_{3}\right), 1.70-1.81\left(4 \mathrm{H}, \mathrm{m}, \mathrm{CH}_{2}\right), 3.74(4 \mathrm{H}$, br.t, $\left.\mathrm{OCH}_{2}\right), 3.79-3.95\left(10 \mathrm{H}, \mathrm{m}, \mathrm{OCH}_{2}, \mathrm{CH}_{3}\right), 4.06(4 \mathrm{H}$, br.t, $\left.\mathrm{CH}_{2} \mathrm{~N}\right), 7.30$ (4H, s, ArH), 7.34 (4H, s, ArH), 7.77 (4H, br.d, NCH, $\mathrm{NCH}), 9.20(2 \mathrm{H}, \mathrm{s}, \mathrm{NCHN}) .{ }^{13} \mathrm{C}$ NMR (100.6 MHz, [D6]DMSO, $298 \mathrm{~K}) \delta_{\mathrm{C}}$ ppm: 145.33, 136.57, 127.89, 127.60, 123.71, 122.27, $68.72,67.70,48.08,35.79,31.28,31.01,30.88,28.99,25.84,25.12$, $22.06,13.89$

5,11,17,23-Tetra-tert-butyl-25,27-ditetradecyl-26,28-bis[4(1-N-methylmorpholinium)butyloxy)-2,8,14,20-tetrathiacalix [4] arene dibromide (11). Reaction time: $31 \mathrm{~h}$. Yield $0.1076 \mathrm{~g} \mathrm{(73 \% ).}$ M.p. $279^{\circ} \mathrm{C}$ (decomp.). Found (\%): C 65.21; H 8.97; N 1.74; S 8.05. $\mathrm{C}_{86} \mathrm{H}_{140} \mathrm{O}_{6} \mathrm{~N}_{2} \mathrm{~S}_{4} \mathrm{Br}_{2}$ requires (\%): C 65.12; $\mathrm{H}$ 8.90; N 1.77; $\mathrm{S} 8.09$. MALDI TOF $m / z: 1503[\mathrm{M}-\mathrm{Br}]^{+}$. IR $(\mathrm{KBr}) v_{\max } \mathrm{cm}^{-1}: 3420\left(\mathrm{C}_{\mathrm{Ar}^{-}}\right.$ $\mathrm{H}), 2922(\mathrm{C}-\mathrm{H}), 1267\left(\mathrm{C}_{-}-\mathrm{O}\right), 1128\left(-\mathrm{CH}_{2}-\mathrm{O}-\mathrm{CH}_{2}-\right) .{ }^{1} \mathrm{H}$ NMR $(400 \mathrm{MHz},[\mathrm{D} 6] \mathrm{DMSO}, 298 \mathrm{~K}) \delta_{\mathrm{H}}$ ppm: $0.86\left(6 \mathrm{H}\right.$, br.s, $\left.\mathrm{CH}_{3}\right), 0.96-$ $1.44\left(84 \mathrm{H}, \mathrm{m}, \mathrm{CMe}_{3}, \mathrm{CMe}_{3}, \mathrm{CH}_{2}\right), 1.56-1.77\left(4 \mathrm{H}, \mathrm{m}, \mathrm{CH}_{2}\right), 3.07-$ $3.65\left(30 \mathrm{H}, \mathrm{m}, \mathrm{CH}_{3}, \mathrm{NH}_{2}, \mathrm{NH}_{2}, \mathrm{CH}_{2}\right), 3.64-4.08\left(16 \mathrm{H}, \mathrm{m}, \mathrm{OCH}_{2}\right.$, $\left.\mathrm{OCH}_{2}, \mathrm{OCH}_{2}\right), 7.34(4 \mathrm{H}, \mathrm{s}, \mathrm{ArH}), 7.41(4 \mathrm{H}, \mathrm{s}, \mathrm{ArH}) .{ }^{13} \mathrm{C} \mathrm{NMR}$ $(100.6 \mathrm{MHz}$, [D6]DMSO, $298 \mathrm{~K}) \delta_{\mathrm{C}} \mathrm{ppm}: 131.67,128.81,128.64$, $126.08,125.03,113.91,67.39,61.52,60.28,59.99,59.89,58.82$, $58.15,51.04,50.82,50.73,45.39,38.06,35.42,31.29,31.17,30.87$, $29.77,28.97,28.70,28.33,23.22,22.36,22.01,20.79,13.85,10.67$.

\section{Results and Discussion}

One of the most commonly used approaches for synthesis of cationic amphiphiles is the quaternization of corresponding tertiary amines or $N$-containing heterocycles by halogen-containing derivatives. It is well known as Menshutkin reaction, which has been named after its discoverer, the Russian chemist Nikolai Menshutkin, who described the procedure in $1890 .^{[27]}$ In this regard, the bromine-containing derivatives are the most attractive building blocks since they are much more reactive in nucleophilic substitution reactions compared to chlorides and cheaper and stable for iodides. The stepwise functionalization of T[4]CA platform with alkyl and bromoalkyl groups in 1,3-alternate stereoisomeric form with following quaternization is a convenient method to obtain a wide series of cationic amphiphiles by simple nucleophilic substitution. It is noteworthy that T[4]CA scaffold adopting 1,3-alternate stereoisomeric form is in a great importance since selective stepwise functionalization of macrocycle lower rim allows to create two molecular domains with quite different properties located on the opposite sides from macrocycle plane. Unlike cone stereoisomeric form, this modification implies only chemical modification of lower rim without anycomplicated modifications of the upper rim. Corresponding bis-alkyl derivatives were obtained earlier using selective functionalization of T[4]CA in Mitsunobu conditions by alkyl moieties on the first step. ${ }^{[20]}$ By the second one bromoalkyl moieties can be easily introduced using Mitsunobu reaction with halohydrines ${ }^{[20]}$ or by alkylation with more commonly used dihalogen derivatives under microwave heating (Scheme 1). ${ }^{[26]}$

To study the influence of steric and electronic effects the reactions of compounds $\mathbf{1}-\mathbf{3}$ with several tertiary amines and $N$-containing heterocycles: triethylamine (TEA), $N, N$ dimethylbenzylamine (DMBA), $\quad N$-methylmorpholine (NMM), pyridine (Py) and $N$-methylimidazole (NMI) were investigated (Scheme 2). Reaction of 1 with TEA was performed in solvents, the most commonly used in nucleophilic substitution: $\mathrm{CHCl}_{3}, \mathrm{CH}_{3} \mathrm{CN}, \mathrm{CH}_{3} \mathrm{COCH}_{3}, \mathrm{THF}$, DMF and solvent-free. According to TLC, reaction did not start even after 120 hours under conventional/microwave heating. Hydrolysis of $\mathbf{1}$ and parent T[4]CA formation was observed in DMF.

Reaction of $\mathbf{1}$ with other aliphatic tertiary amines - DMBA and NMM was also unsuccessful. And only interaction with heterocycles NMI and Py leads to the desirable products $\mathbf{4}$ and $\mathbf{5}$ with high yields (Table 1). Such structure effect of $N$-containing compounds on their reactivity in $\mathrm{S}_{\mathrm{N}} 2$ reactions was quite unexpected because the reverse order of reactivity was usually observed in the case of halogenoalkanes. ${ }^{[28,29]}$ For example, TEA is significantly more active that DMBA and Py in the reaction with 


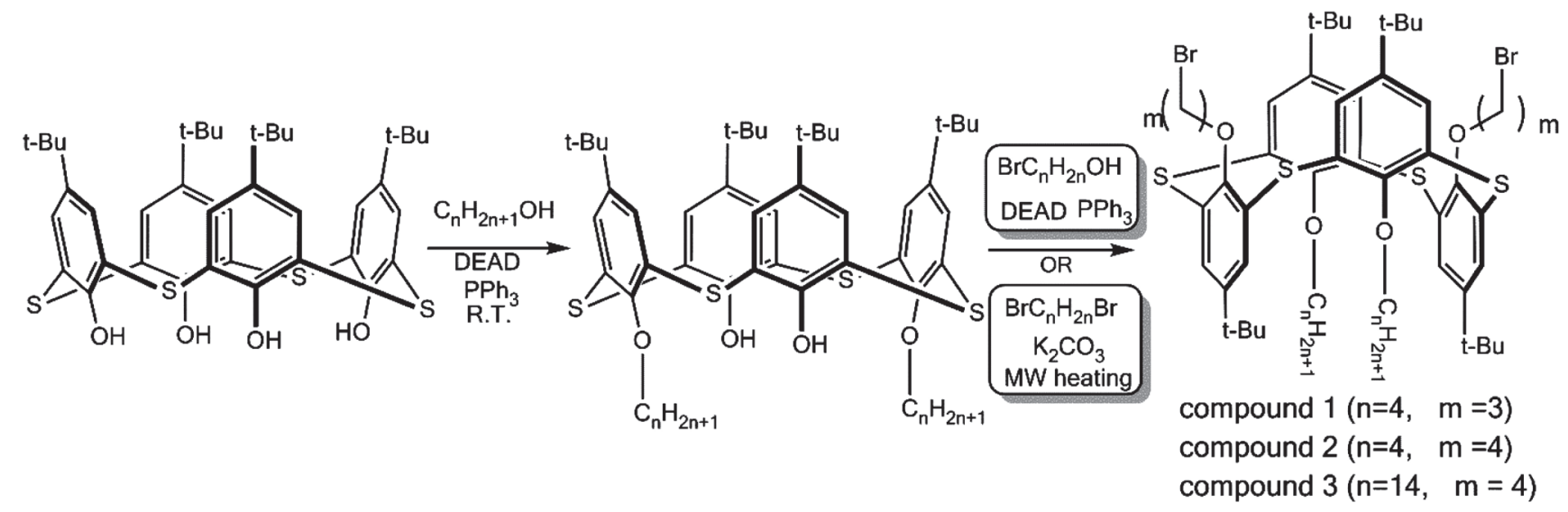

Scheme 1. General pathways for the synthesis of bifunctional T[4]CA derivatives containing alkyl and bromoalkyl moieties.

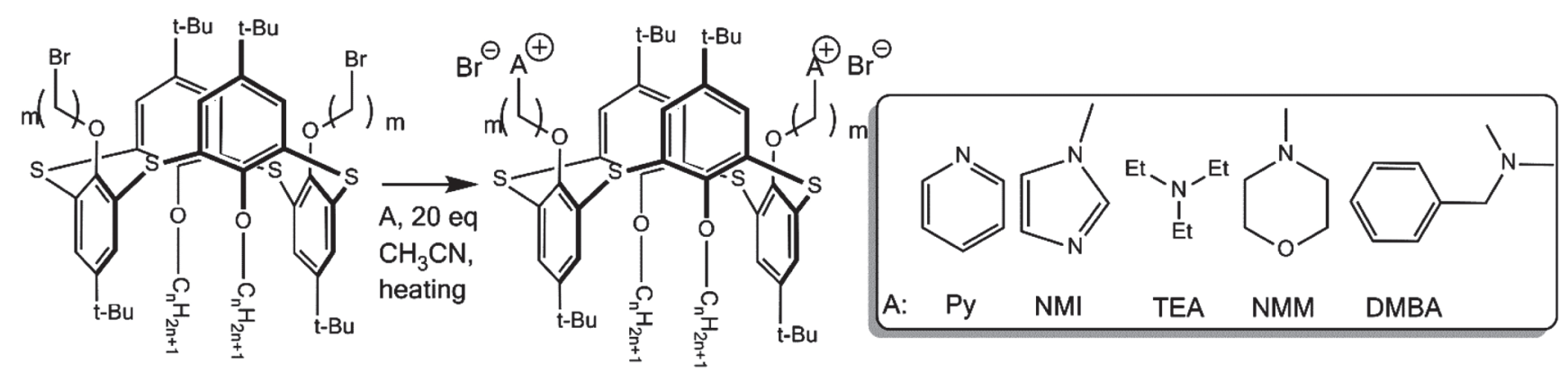

Scheme 2. Alkylation of tertiary amines and $N$-heterocycles by T[4]CA derivatives 1-3.

Table 1. Reaction of 1-3 with $N$-nucleophiles: times of reaction and yields of corresponding products*.

\begin{tabular}{cccccc}
\hline Compound & Nucleophile & $\mathrm{n}$ & $\mathrm{m}$ & Time, $\mathrm{h}$ & Yield, \% \\
\hline $\mathbf{4}$ & Py & 4 & 3 & 37 & 88 \\
$\mathbf{5}$ & NMI & 4 & 3 & 34 & 87 \\
$\mathbf{6}$ & Py & 4 & 4 & 12 & 84 \\
$\mathbf{7}$ & NMI & 4 & 4 & 10 & 85 \\
$\mathbf{8}$ & NMM & 4 & 4 & 45 & 81 \\
$\mathbf{9}$ & Py & 14 & 4 & 25 & 79 \\
$\mathbf{1 0}$ & NMI & 14 & 4 & 23 & 75 \\
$\mathbf{1 1}$ & NMM & 14 & 4 & 31 & 73 \\
\hline
\end{tabular}

*Reaction conditions: 20 eq. of amine, $\mathrm{CH}_{3} \mathrm{CN}, 130{ }^{\circ} \mathrm{C}$

methyl iodide. Values of $\lg k_{2}$ are $-2.73,-3.88$ and -4.17 , corresponingly. ${ }^{[28]}$ Similar dependence was also observed for benzyl halogenates: TEA is an order of magnitude more active than Py ${ }^{[29]}$ Such reactivity changes are in line with the basicity decrease $\left(\lg K_{\mathrm{b}}\right)$ : TEA $(3.13)^{[30]}>$ DMBA $(4.97)$ ${ }^{[31]}>\mathrm{Py}(8.85) .{ }^{[30]}$ What is the reason of the reactivity inversion in the case of macrocycle $\mathbf{1}$ ?

It is well known that $\mathrm{S}_{\mathrm{N}} 2$ reactions are very sensitive to the steric hindrances, which affect the nucleophile's strength. ${ }^{[32]}$ For example, the rate constant of methyl iodine with trimethylamine is about 100 times greater with tri- ethylamine owing to the steric effect of the ethyl group. ${ }^{[29]}$ Therefore, unusual low reactivity of more nucleophilic among investigated nucleophiles TEA may be associated with steric hindrances both from bulky tert-butyl groups of 1 and ethyl fragments of TEA. Earlier, ${ }^{[20]}$ we have demonstrated that the steric hindrances of bulky tertbutyl groups are the main reason of the slow bromine substitution by azide anion in bromopropyl thiacalixarene derivatives in 1,3-alternate stereoisomeric form. Optimized

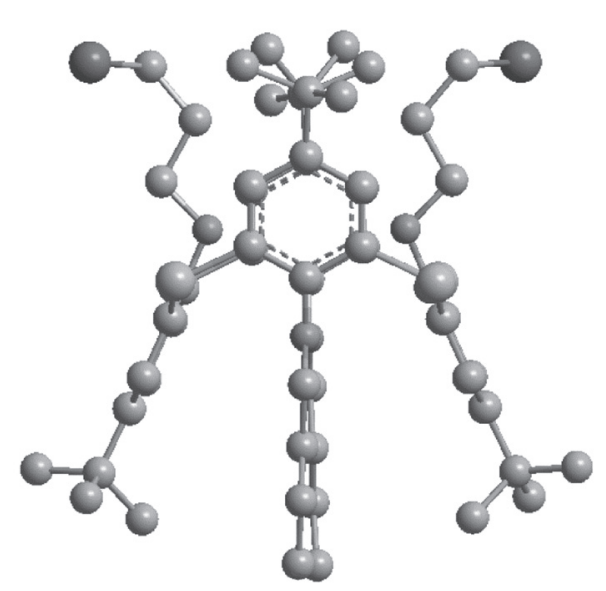

Figure 1. The conformation of compound 1 in global energy minimum (hydrogen atoms are hidden for simplicity, alkyl chain length is limited to five carbon atoms). 
structure of $\mathbf{1}$, calculated by PRIRODA 11 program (method DFT, functional PBE, basis $3 z)^{[33]}$ is presented in Figure 1.

As can be seen from Figure 1, the location of $\mathrm{C}-\mathrm{Br}$ bonds results in poor accessibility of carbon atom for the backside attack of nucleophile due to the bulky tert-butyl groups. Nevertheless, small azide anion reacts with bromopropyl calixarene under microwave heating. Tertiary aliphatic amines like TEA, NMM and DMBA in their sizes and steric hindrances significantly exceed the linear azide anion. Thus, it becomes evident that reaction behavior of tertiary amines with $\mathbf{1}$ is the result of steric hindrances. Less nucleophilic NMI and Py exhibit higher reactivity due to their planar compact structures.

To evaluate steric accessibility of nitrogen atoms in the investigated nucleophiles a Topological Steric Effect Index (TSEI) of a group based on the relative specific volume of the reaction centre screened by the atoms of the substituents is used. ${ }^{[34]}$ The TSEI value of the molecule can be easily calculated using ChemAxon JChem Topological Analysis Plugin ${ }^{[35]}$ and has a clear physical meaning. Calculated TSEI values for investigated amines are presented in Table 2.

Table 2. TSEI values of the investigated $N$-nucleophiles.

\begin{tabular}{cccccc}
\hline Amine & TEA & DMBA & NMM & NMI & Py \\
\hline TSEI & 1.12 & 1.11 & 1.12 & 1.04 & 1.01 \\
\hline
\end{tabular}

Indeed, the highest TSEI values are obtained for the most branched TEA, DMBA and NMM, while heterocyclic NMI and Py have the smallest ones. This TSEI series is in full agreement with the observed reactivity. Therefore, steric factor is governed the reactivity of investigated $N$-containing nucleophiles.
This conclusion was confirmed by the reactions of compounds $\mathbf{2}$ and $\mathbf{3}$, in which the spacer between the macrocycle and bromine was increased to four methylene groups (Scheme 1). The reactivity of calixarene substantially increases going from three methylene groups in $\mathbf{1}$ to four in compound $\mathbf{2}$. Reaction with NMI and Py finished after 10-12 hours giving corresponding quaternary compounds 6 and 7 in high yields, and even NMM gives product 8 after 45 hours of heating. Such increase of reactivity is in a good accordance with increased steric accessibility of carbon atom for nucleophilic substitution due to small rise above calixarene tert-butyl groups. With regard to NMM decrease of the freedom degree in the cycle results in its reactivity compare to DMBA and TEA despite of similar TSEI values. The presence of long alkyl fragments in compound $\mathbf{3}$ leads to two-fold increase of reaction times and insignificant decrease of yields in compounds 9-11.

All compounds were characterized by 1- and 2-D NMR techniques, MALDI-TOF spectrometry, IR spectroscopy and elemental analysis. According to 2D NOESY spectra of compounds (Figure 2, exemplified by compound 5) there are cross-peaks between signals of $\mathrm{CH}_{2} \mathrm{O}$ protons $7(\delta=3.90 \mathrm{ppm})$ of propylene linker and aromatic protons $8^{\prime}(\delta=7.33 \mathrm{ppm})$ as well as methylene 10 protons $(\delta=3.75 \mathrm{ppm})$ of butoxy fragments and another aromatic protons 8 of thiacalixarene $(\delta=7.36 \mathrm{ppm})$. It indicates clearly on the 1,3-alternate stereoisomeric form of thiacalixarene. Besides this, there are a lot of cross peaks of imidazolyl protons with surrounding environment. Thus, there are crosspeaks between signals of imidazolyl protons 4 and $2(\delta=7.66$ and $9.25 \mathrm{ppm}$ ) with methylene protons of propylene linker 5,6 and $7(\delta=4.26,1.50,3.90 \mathrm{ppm})$. It is noteworthy that imidazolyl proton 3 has no cross-peaks with methylene protons of linker with three methylene groups indicating
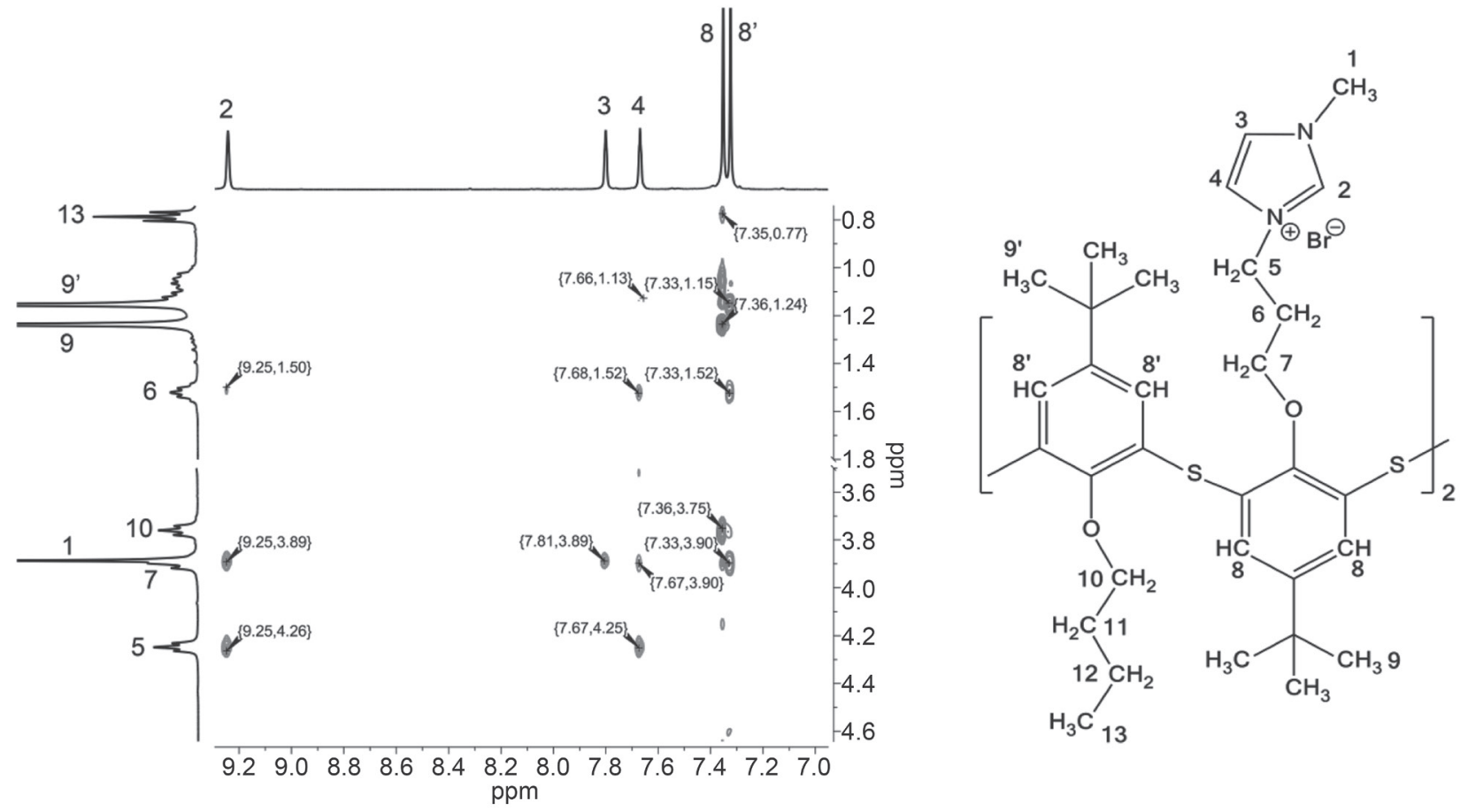

Figure 2. 2D NOESY NMR spectra ([D6]DMSO, $298 \mathrm{~K}$ ) of compound 5. 
thus distance from it. Simultaneously, there are cross-peaks between signals of imidazolyl protons 3 and $2(\delta=7.81$ and $9.25 \mathrm{ppm})$ with signal of methyl protons $1(\delta=3.89 \mathrm{ppm})$, indicating thus quaternization on the third position of heterocycle.

Therefore, quaternization of amines by calixarenes 1-3 is strongly dependent from steric factor. In the case of calixarene $\mathbf{1}$ bulky tert-butyl groups have a strong influence on reactivity of calixarene resulting in high times of reaction preventing thus reaction in the case of bulky TEA, NMM and DMBA. Increase of linker from three to four methylene groups results in increased reactivity due to the reduced sterical hindrances. Less nucleophilic amines NMI and Py are more reactive for their planar compact structure, which is reflected in their TSEI values. In this way, the valuation of TSEI values is very convenient way for $\mathrm{S}_{\mathrm{N}} 2$ reactivity assessment in molecules with hard accessible reaction center.

\section{Conclusions}

A series of new cationic amphiphilic $p$-tert-butylthiacalix [4] arene derivatives adopting 1,3-alternate stereoisomeric form was synthesized by simple quaternization of tertiary amines by bifunctional thiacalixarenes containing alkyl and bromoalkyl moieties of various length. It was found that reaction can be implemented only with sterically accessible tertiary amines, and aromatic $N$-containing heterocycles are the most reactive. In turn, the reactivity of thiacalixarene bromine derivatives dramatically falls going from bromobutyl to bromopropyl substituents due to the increasing sterical hindrances from tert-butyl groups. Thus, alkylation of thiacalixarene bromine derivatives in 1,3-alternate is sterically controlled. It was shown that calculated topological steric effect index (TSEI) of $N$-nucleophiles is in an accordance with their reactivity. Therefore, the valuation of TSEI values is very convenient way for prediction of sterically controlled $\mathrm{S}_{\mathrm{N}} 2$ reactions.

Acknowledgements. We thank the Russian Science Foundation for the financial support of this work (grant № 14-13-01151).

\section{References}

1. Lombardo D., Kiselev M.A., Magazù S., Calandra P. Adv. Condens. Matter Phys. 2015, 2015, 1-22.

2. Voskuhl J., Ravoo B.J. Chem. Soc. Rev. 2009, 38, 495-505.

3. Jie K., Zhou Y., Yao Y., Huang F. Chem. Soc. Rev. 2015, 44, 3568-3587.

4. Thambi T., Park J.H., Lee D.S. Biomater. Sci. 2016, 4, 55-69.

5. Tschiche A., Malhotra S., Haag R. Nanomedicine 2014, 9, 667-93.
6. Wenz G., Han B.H., MSller A. Chem. Rev. 2006, 106, 782-817.

7. Lagona J., Mukhopadhyay P., Chakrabarti S., Isaacs L. Angew. Chem. 2005, 117, 4942-4949.

8. Wang K., Yang Y.-W., Zhang S.X.-A. Chem.J. Chinese U. 2012, 33, 1-13.

9. Asfari Z., Bohmer V., Harrowfield J., Vicens J. Calixarenes 2001. Netherland: Springer Netherlands, 2001. 684 p.

10. Kumar R., Lee Y.O., Bhalla V., Kumar M., Kim J.S. Chem. Soc. Rev. 2014, 43, 4824-4870.

11. Giuliani M., Morbioli I., Sansone F., Casnati A. Chem. Commun. 2015, 51, 14140-14159.

12. Tauran Y., Coleman A.W., Perret F., Kim B. Curr. Org. Chem. 2015, 19, 2250-2270.

13. Shinkai S., Mori S., Koreishi H., Tsubaki T., Manabe O. J. Am. Chem. Soc. 1986, 108, 2409-2416.

14. Perret F., Lazar A.N., Coleman A.W. Chem. Commun. 2006, 23, 2425-2438.

15. Arduini A., Pochini A., Reverberi S., Ungaro R. J. Chem. Soc., Chem. Commun. 1984, 15, 981-982.

16. Rodik R.V., Anthony A.-S., Kalchenko V.I., Mély Y., Klymchenko A.S. New J. Chem. 2015, 39, 1654-1664.

17. Zadmard R., Schrader T. J. Am. Chem. Soc. 2005, 127, 904-915.

18. Ukhatskaya E., Kurkov S., Matthews S., Loftsson Th. J. Pharm. Sci. 2013, 102, 3485-3512.

19. Mourer M., Dibama H.M., Fontanay S., Grare M., Duval R.E., Finance Ch., Regnouf-de-Vains J.-B. Bioorg. Med. Chem. 2009, 17, 5496-5509.

20. Burilov V.A., Nugmanov R.I., Ibragimova R.R., Solovieva S.E., Antipin I.S. Mendeleev Commun. 2015, 25, 177-179.

21. Burilov V., Mironova D.A., Ibragimova R.R., Solovieva S.E., Antipin I.S. Bionanoscience 2016, 6, 427-430.

22. Ibragimova R.R., Burilov V.A., Aymetdinov A.R., Mironova D.A., Evtugyn V.G., Osin Yu.N., Solovieva S.E., Antipin I.S. Macroheterocycles 2016, 9, 433-441.

23. Burilov V.A., Mironova D.A., Ibragimova R.R., Nugmanov R.I., Solovieva S.E., Antipin I.S. Colloids Surf., A 2017, 515, $41-49$.

24. Armarego W.L.F., Chai C.L.L. Purification of Laboratory Chemicals. New York: Elsevier, 2009. $760 p$.

25. Iki N., Kabuto C., Fukushima T., Kumagai H., Takeya H., Miyanari S., Miyashi T., Miyano S. Tetrahedron 2000, 56, 1437-1443.

26. Burilov V.A., Ibragimova R.R., Gafiatullin B.H., Solovieva S.E., Antipin I.S. Butlerov Commun. 2016, 47(8), 23-28.

27. Menschutkin N. Phys. Chem. 1980, 5, 589-600.

28. Tables of Rate and Equilibrium Constants of Heterolytic Organic Reactions, Vol. 4 (Palm V.A., Ed.). Moscow: VINITI, 1977. 618 p. (in Russ.) [Таблиць констант скорости и равновесия гетеролитических органических реакичй, T. 4 (Пальм В.А., ред.). М.: ВИНИТИ, 1977. 618 с.].

29. Wang T.-T., Huang T.-Ch. Chem. Eng. J. 1993, 53, 107-113.

30. Kuna S., Pawlaic Z., Tusk M. J. Chem. Soc., Faraday Trans. 1982, 78, 2685-2692.

31. Frenna V., Vivona N., Consiglio G., Spinelli D. J. Chem. Soc., Perkin Trans. 2 1985, 1865-1868.

32. Katritzky A.R., Brycki B.E. Chem. Soc. Rev. 1990, 19, 83-105.

33. Laikov D.N. Chem. Phys. Lett. 2005, 416, 116-120.

34. Cao Ch., Liu L. J. Chem. Inf. Comput. Sci. 2004, 44, 678-687.

35. http://www.chemaxon.com. 